doi:10.13108/2021-13-4-80

\title{
ON COEFFICIENT MULTIPLIERS FOR AREA PRIVALOV CLASSES
}

\section{E.G. RODIKOVA}

\begin{abstract}
The problem of describing the Taylor coefficients of functions analytic in a disk was first resolved for the Nevanlinna class by an outstanding Soviet mathematician S.N. Mergelyan in the beginning of 20th century. Later, the studies devoted to obtaining similar estimates in various classes of analytic functions were made by known Russian and foreign specialists in the complex analysis: G. Hardy, J. Littlewood, A.A. Friedman, N. Yanagihara, M. Stoll, S.V. Shvedenko and others.

In the paper we introduce a area Privalov class $\tilde{\Pi}_{q},(q>0)$, being a generalization of a known area Nevanlinna class. In the first part of the paper we obtain a sharp estimate for the growth of an arbitrary function in the area Privalov class, we describe the Taylor coefficients for this function. In the second part of the work, on the base of the obtained estimates we describe completely the coefficient multipliers from area Privalov classes into the Hardy classes. In a simplified form this problem can be formulated as follows: by what factors the Taylor coefficients of a function in a given class $\tilde{\Pi}_{q}, q>0$, should be multiplied in order to get the Taylor coefficients of a function in a Hardy class.
\end{abstract}

Keywords: area Privalov class, Taylor coefficients, multiplier, growth, analytic functions.

Mathematics Subject Classification:30H50, 30H10, 30H15

\section{INTRODUCTION}

Let $\mathbb{C}$ be the complex plane, $D$ be the unit disk in $\mathbb{C}$ and $H(D)$ be the set of all functions analytic in $D$. For all $0<q<+\infty$ we define a Privalov class $\Pi_{q}$ :

$$
\Pi_{q}=\left\{f \in H(D): \sup _{0<r<1} \frac{1}{2 \pi} \int_{-\pi}^{\pi}\left(\ln ^{+}\left|f\left(r e^{i \theta}\right)\right|\right)^{q} d \theta<+\infty\right\}
$$

where $\ln ^{+} a=\max (\ln a, 0)$ for each $a>0$.

First the classes $\Pi_{q}$ were considered by I.I. Privalov in [4]. As $q=1$, the Privalov class coincides with a well-known in a scientific literature class of functions of bounded type or the Nevanlinna class $N$ [2]. Employing Hölder inequality, it is easy to prove the chain of inclusions:

$$
\Pi_{q}(q>1) \subset N \subset \Pi_{q}(0<q<1) .
$$

For $q>1$ the class $\Pi_{q}$ was studied by foreign mathematicians M. Stoll, M. Pavlović, M. Jevtić. R. Meštrović and Russian specialists on the theory of functions V.I. Gavrilov, A.V. Subbotin, D.A. Efimov, see [1] and the references therein. The case $0<q<1$ was studied in the works by the author of the present paper and also by F.A. Shamoyan and his co-authors, see [8]-[10], [14], [16], [23]-[25].

For all $0<q<+\infty$ we introduce one more class

$$
\tilde{\Pi}_{q}=\left\{f \in H(D): \int_{0}^{1} \int_{-\pi}^{\pi}\left(\ln ^{+}\left|f\left(r e^{i \theta}\right)\right|\right)^{q} d \theta d r<+\infty\right\} .
$$

E.G. Rodikova, On coefficient multipliers for area Privalov classes.

(C) Rodikova E.G. 2021.

Submitted January 31, 2021. 
We call it a area Privalov class or Privalov class by area. As $q=1$, the area Privalov class coincides with a well-known area Nevanlinna class

$$
\mathbf{N}=\left\{f \in H(D): \int_{0}^{1} \int_{-\pi}^{\pi} \ln ^{+}\left|f\left(r e^{i \theta}\right)\right| d \theta d r<+\infty\right\}
$$

or

$$
\mathbf{N}=\left\{f \in H(D): \iint_{D} \ln ^{+}|f(z)| d x d y<+\infty\right\} . \quad z=x+i y,
$$

The area Nevanlinna class is involved in the scale of the Nevanlinna-Djrbashian classes $N_{\alpha}$ :

$$
N_{\alpha}=\left\{f \in H(D): \int_{0}^{1}(1-r)^{\alpha} T(r, f) d r<+\infty\right\}, \quad \alpha>-1,
$$

where $T(r, f)$ is the Nevanlinna characteristics of the function $f \in H(D)$, see [2]:

$$
T(r, f)=\frac{1}{2 \pi} \int_{-\pi}^{\pi} \ln ^{+}\left|f\left(r e^{i \theta}\right)\right| d \theta, \quad 0<r<1 .
$$

In their turn, the classes $N_{\alpha}$ are involved the scale of classes $S_{\alpha}^{q}$ :

$$
S_{\alpha}^{q}=\left\{\int_{0}^{1}(1-r)^{\alpha} T^{q}(r, f) d r<+\infty\right\}, \quad \alpha>-1, \quad 0<q<+\infty .
$$

The classes $S_{\alpha}^{q}$ were introduced and studied in [12] by F.A. Shamoyan.

Employing Hölder inequality, it is easy to show that

$$
\tilde{\Pi}_{q} \subset S_{0}^{q} \quad \text { as } \quad q>1, \quad \tilde{\Pi}_{q} \supset S_{0}^{q} \quad \text { as } \quad 0<q<1 .
$$

We note that the classes $\tilde{\Pi}_{q}$ naturally arise in studying integro-differential operators in the Privalov space. In a recent joint work [25], the author of the present and F.A. Shamoyan paper proved that the Privalov class is not invariant with respect to the differentiation operator for all $q>0$, that is, the Bloch-Nevanlinna conjecture fails in the Privalov spaces. It was also established in [25] that the derivative of an arbitrary function with no zeroes from the Privalov class $\Pi_{q}$ belongs to the Privalov class $\tilde{\Pi}_{q}$ by area.

In the present work we obtain sharp estimates for the maximum of the absolute value and the Taylor coefficients of the functions in the classes $\tilde{\Pi}_{q}, q>0$, see Section 2, and on this base in Section 3 we describe coefficient multipliers from the are Privalov classes $\tilde{\Pi}_{q}, q>0$, into the Hardy classes $H^{p}, 0<p \leqslant+\infty$.

We note that the problem on describing the Taylor coefficients of the functions analytic in a disk was first resolved by an outstanding Soviet mathematician S.N. Mergelyan in the beginning of 20th century, see [5]. An analog of Mergelyan's result in the Hardy classes in a disk was proved by G. Hardy and J. Littlewood, A. Friedman [28], in the area Nevanlinna classes by S.V. Shvedenko [18], in the area Privalov classes $\Pi_{q}$ for all $q>1$ sharp estimates for the growth of a function and its Taylor coefficients were established by M. Stoll in [26] and for $0<q<1$ this was done by the author of the present paper in [23].

Estimating of the Taylor coefficient is closely related with describing the coefficient multipliers in the Privalov classes. As it was mentioned in [1], in a simplified form the problem is formulated as follows: by which multipliers one should multiply the Taylor coefficients of the functions in a given class in order them to acquire prescribed properties, for instance, to make them bounded or to form an absolutely converging series. Postulating the obtained products to be the Taylor coefficients of the functions in some other class, we arrive at a general definition of the coefficient multiplier. 
Definition 1.1. Let $X$ and $Y$ be some classes of the functions analytic in the unit disk D. A sequence of complex numbers $\Lambda=\left\{\lambda_{k}\right\}_{k=1}^{+\infty}$ is called a coefficient multiplier from the class $X$ into the class $Y$ if for an arbitrary function $f \in X, f(z)=\sum_{k=0}^{+\infty} a_{k} z^{k}$, the function $\Lambda(f)(z)=\sum_{k=0}^{+\infty} \lambda_{k} a_{k} z^{k}$ belongs to $Y$. This situation is shortly denoted by $C M(X, Y)$.

Many works by Russian and foreing mathematicians were devoted to describing the multipliers in various classes of holomorphic functions; we mention some of them [1], [3], [15], [17], [23], [27].

\section{Estimate FOR GROWTH AND TAYLOR COEFFICIENTS OF FUNCTIONS FROM AREA PRIVALOV CLASSES}

Throughout the paper, unless else is stated, we suppose that $q>0$. By $c, c_{1}, \ldots, c_{n}(\alpha, \beta, \ldots)$ we denote positive constants independent of $\alpha, \beta, \ldots$

The following statement holds true.

Theorem 2.1. If $f \in \tilde{\Pi}_{q}$, then

$$
\ln ^{+} M(r, f)=o\left((1-r)^{-\frac{2}{q}}\right), \quad r \rightarrow 1-0,
$$

where $M(r, f)=\max _{|z|=r}|f(z)|$.

Proof. We choose an arbitrary point $z_{0} \in D$ and we denote

$$
K_{z_{0}}=\left\{\zeta \in D:\left|\zeta-z_{0}\right|<\frac{1}{2}\left(1-\left|z_{0}\right|\right)\right\}
$$

where $d m_{2}$ is the area Lebesgue measure. By estimate [22, Thm. 9.1.1, Est. (9.3)]

$$
\left(\ln ^{+}\left|f\left(z_{0}\right)\right|\right)^{q} \leqslant \frac{c(q)}{\left(1-\left|z_{0}\right|\right)^{2}} \int_{K_{z_{0}}}\left(\ln ^{+}|f(\zeta)|\right)^{q} d m_{2}(\zeta)
$$

we obtain:

$$
\left(\ln ^{+}\left|f\left(z_{0}\right)\right|\right)^{q} \leqslant \frac{c(q)}{\left(1-\left|z_{0}\right|\right)^{2}} \int_{-\pi}^{\pi} \int_{\left|z_{0}\right|-\frac{1-\left|z_{0}\right|}{2}}^{\left|z_{0}\right|+\frac{1-\left|z_{0}\right|}{2^{2}}}\left(\ln ^{+}\left|f\left(\rho e^{i \theta}\right)\right|\right)^{q} d \rho d \theta
$$

and this yields:

$$
\left(\ln ^{+}\left|f\left(z_{0}\right)\right|\right)^{q} \leqslant \frac{c(q)}{\left(1-\left|z_{0}\right|\right)^{2}} \int_{-\pi}^{\pi} \int_{0}^{1}\left(\ln ^{+}\left|f\left(\rho e^{i \theta}\right)\right|\right)^{q} d \rho d \theta .
$$

The latter inequality implies estimate (2.1). The proof is complete.

Theorem 2.2. If $f(z)=\sum_{k=0}^{+\infty} a_{k} z^{k}$ is the Taylor series of the function $f \in \tilde{\Pi}_{q}$, then

$$
\ln ^{+}\left|a_{k}\right|=o\left(k^{\frac{2}{2+q}}\right), \quad k \rightarrow+\infty .
$$

Proof. It follows from the Cauchy inequality and estimate 2.1) in Theorem 2.1 that for an arbitrary small $\varepsilon>0$ there exists $r_{\varepsilon} \in(0,1)$ such that

$$
\left|a_{k}\right| \leqslant r^{-k} \exp \left(\varepsilon(1-r)^{-\frac{2}{q}}\right), \quad r_{\varepsilon}<r<1, \quad k=0,1, \ldots,
$$

which is equivalent to

$$
\ln ^{+}\left|a_{k}\right| \leqslant \varepsilon(1-r)^{-\frac{2}{q}}-k \ln r, \quad r_{\varepsilon}<r<1, \quad k=0,1, \ldots
$$


We introduce a function

$$
\phi(r)=\varepsilon(1-r)^{-\frac{2}{q}}-k \ln r .
$$

Let us find its infimum. We calculate its derivative:

$$
\phi^{\prime}(r)=\frac{2 \varepsilon}{q} \frac{1}{(1-r)^{\frac{2}{q}+1}}-\frac{k}{r} .
$$

We find the minimum of the function $\phi(r)$ by solving the equation $\phi^{\prime}(r)=0$ :

$$
\frac{2 \varepsilon}{q} \frac{r}{(1-r)^{\frac{2}{q}+1}}=k .
$$

Since the function in the left hand side of this identity increases and is injective, then this equation is uniquely solvable in the interval $(0,1)$. We denote the point of the minimum of the function $\phi(r)$ by $r_{k}$.

Let us consider the case $0<q<1$. For the sake of convenience we introduce the following notations:

where $\delta>1$.

$$
t_{k}=\frac{1}{\delta \sqrt{r_{k}}}, \quad s_{k}=\frac{1-r_{k}}{\delta \sqrt{r_{k}}},
$$

We can suppose that $s_{k}<t_{k} \leqslant 1$. Indeed, the inequality $s_{k}<t_{k}$ is obvious. Then $t_{k} \leqslant 1$ is equivalent to

$s_{k}<1$ is equivalent to

$$
\sqrt{r_{k}} \geqslant \frac{1}{\delta}
$$

$$
\sqrt{r_{k}}>\frac{\sqrt{\delta^{2}+4}-\delta}{2}
$$

and 2.6 implies 2.7$)$.

In terms of new notations equation (2.5) becomes

or

$$
\frac{2 \varepsilon}{q \delta^{2}} \frac{1}{s_{k}^{2}}\left(\frac{t_{k}}{s_{k}}\right)^{\frac{2}{q}-1}=k
$$

$$
\frac{s_{k}^{\frac{2}{q}+1}}{t_{k}^{\frac{2}{q}-1}}=\frac{2 \varepsilon}{k q \delta^{2}} .
$$

Since $t_{k} \leqslant 1$, the latter identity implies the estimate

$$
s_{k} \leqslant\left(\frac{2 \varepsilon}{k q \delta^{2}}\right)^{\frac{1}{q}+1} .
$$

By the same identity we obtain:

$$
\left(\frac{t_{k}}{s_{k}}\right)^{\frac{2}{q}}=\left(\frac{k s_{k}^{2} q \delta^{2}}{2 \varepsilon}\right)^{\frac{2}{2-q}}
$$

Taking into consideration estimate (2.8), we obtain:

$$
\left(\frac{t_{k}}{s_{k}}\right)^{\frac{2}{q}} \leqslant\left(\frac{q \delta^{2}}{2 \varepsilon}\right)^{\frac{2}{2+q}} k^{\frac{2}{2+q}} .
$$

Employing established estimates (2.8), (2.9), we estimate the value of the function $\phi(r)$ at the point $r=r_{k}$ of its strict minimum:

$$
\phi\left(r_{k}\right)=\varepsilon\left(1-r_{k}\right)^{-\frac{2}{q}}-k \ln r_{k}
$$


Taking into consideration estimate $(2.9)$, we now get:

$$
\phi\left(r_{k}\right) \leqslant \varepsilon\left(\frac{q \delta^{2}}{2 \varepsilon}\right)^{\frac{2}{2+q}} k^{\frac{2}{2+q}}-k \ln r_{k}
$$

In order to estimate the latter term, we observe that

$$
\frac{\left(r_{k}\right)^{-\frac{1}{2}}-r_{k}^{\frac{1}{2}}}{2}=\frac{\exp \left(-\frac{1}{2} \ln r_{k}\right)-\exp \left(\frac{1}{2} \ln r_{k}\right)}{2}=-\sinh \left(\frac{1}{2} \ln r_{k}\right)=\sinh \left(-\frac{1}{2} \ln r_{k}\right)=\frac{s_{k} \delta}{2}
$$

and this implies

$$
-\ln r_{k}=2 \operatorname{arcsinh} \frac{s_{k} \delta}{2} \leqslant 2 \frac{s_{k} \delta}{2}, \quad-k \ln r_{k} \leqslant k s_{k} \delta
$$

Thus, we have:

$$
\phi\left(r_{k}\right) \leqslant k^{\frac{2}{2+q}} \varepsilon^{\frac{q}{2+q}}\left(q \delta^{2}\right)^{\frac{2}{2+q}} \cdot\left(2+\frac{1}{q \delta}\right) .
$$

This implies needed estimate 2.2.

We proceed to the case $q>1$. Here for the sake of convenience we introduce the following notations:

In this case $s_{k} \leqslant 1 \leqslant t_{k}$.

$$
t_{k}=\frac{1}{\sqrt{r_{k}}}, \quad s_{k}=\frac{1-r_{k}}{\sqrt{r_{k}}} .
$$

In terms of new notations equation $(2.5)$ becomes:

$$
\frac{2 \varepsilon}{q} \cdot \frac{1}{t_{k}^{2}} \cdot\left(\frac{t_{k}}{s_{k}}\right)^{\frac{2}{q}+1}=k
$$

which is equivalent to

$$
s_{k}^{\frac{2}{q}+1}=\frac{2 \varepsilon}{q k} \frac{t_{k}}{t_{k}^{2\left(1-\frac{1}{q}\right)}} .
$$

This yields:

$$
s_{k} \leqslant \frac{2 \varepsilon}{q k} t_{k} \leqslant \frac{2 \varepsilon}{q k} \sqrt{s_{k}^{2}+2}
$$

We finally obtain the following estimate for $s_{k}$ :

$$
s_{k} \leqslant\left(\frac{2 \sqrt{3} \varepsilon}{k q}\right)^{\frac{1}{q}+1}
$$

Further arguing follow the same lines as in the case $0<q<1$. The proof is complete.

\section{DESCRIPTION OF COEFFICIENT MULTIPLIERS FROM AREA PRIVALOV CLASSES INTO HARDY CLASSES}

For all values of the parameter $0<p<+\infty$ we introduce the Hardy classes in a disk:

$$
H^{p}:=\left\{f \in H(D): \sup _{0<r<1} \int_{-\pi}^{\pi}\left|f\left(r e^{i \varphi}\right)\right|^{p} d \varphi<+\infty\right\},
$$

where $H^{\infty}$ is the class of bounded analytic in $D$ functions.

In this section we describe coefficient multipliers acting from the area Privalov classes into the Hardy classes. The following theorem holds true. 
Theorem 3.1. Let $\Lambda=\left\{\lambda_{k}\right\}_{k=1}^{+\infty}, q>0,0<p \leqslant+\infty$. The identity $\Lambda=C M\left(\tilde{\Pi}_{q}, H^{p}\right)$ holds true if and only if

$$
\left|\lambda_{k}\right|=O\left(\exp \left(-c k^{\frac{2}{2+q}}\right)\right), \quad k \rightarrow+\infty, \quad c>0 .
$$

The proof of this theorem is based on a series of auxiliary lemmata.

Lemma 3.1. [1, Lm. 9.7] Let $F$ and $H$ be linear classes of functions holomorphic in the unit disk $D$ with metrics, the convergence with respect to which is not weaker than the uniform convergence on the compact sets $D$. Then each coefficient multiplier from the class $F$ into the class $H$ is linear and closed as an operator between linear and metric spaces $F$ and $H$.

In order to formulate a next lemma, in the class $\tilde{\Pi}_{q}$ we introduce a metrics by the rule:

$$
\begin{aligned}
& \rho(f, g)=\int_{0}^{1} \int_{-\pi}^{\pi} \ln ^{q}\left(1+\left|f\left(r e^{i \theta}\right)-g\left(r e^{i \theta}\right)\right|\right) d \theta d r, \quad 0<q<1, \\
& \rho(f, g)=\left(\int_{0}^{1} \int_{-\pi}^{\pi} \ln ^{q}\left(1+\left|f\left(r e^{i \theta}\right)-g\left(r e^{i \theta}\right)\right|\right) d \theta d r\right)^{\frac{1}{q}}, \quad q>1 .
\end{aligned}
$$

Lemma 3.2. The class $\tilde{\Pi}_{q}$ with the introduced metrics is an F-space.

Proof. Let $0<q<1$, the case $q>1$ can be proved in the same way.

The proof is equivalent to checking following properties of the metrics, cf. [11].

a) $\rho(f, g)=\rho(f-g, 0)$, which is obvious;

b) $\tilde{\Pi}_{q}$ is a complete metric space.

Let $\left\{f_{n}\right\}$ be an arbitrary fundamental sequence in the class $\tilde{\Pi}_{q}$, that is, for each $\varepsilon>0$ there exists an index $N(\varepsilon)>0$ such that for all $n, m>N$ the inequality $\rho\left(f_{n}, f_{m}\right)<\varepsilon$ holds. Let us show that it converges to some function $f \in \tilde{\Pi}_{q}$. We observe that the functions $\ln \left(1+\left|f_{n}\right|\right)$ are subharmonic in D. Employing again the estimate from [22, Thm. 9.1.1], we obtain:

$$
\ln ^{q}\left(1+\left|f_{n}\left(R e^{i \theta}\right)-f_{m}\left(R e^{i \theta}\right)\right|\right) \leqslant \frac{c(q)}{(1-R)^{2}} \cdot \rho\left(f_{n}, f_{m}\right)
$$

and hence,

$$
\left|f_{n}\left(r e^{i \theta}\right)-f_{m}\left(r e^{i \theta}\right)\right| \rightarrow 0, \quad n, m \rightarrow+\infty,
$$

for all $0<r<R<1, \theta \in[-\pi, \pi]$. Thus, the fundamental sequence $\left\{f_{n}\right\} \in \tilde{\Pi}_{q}$ converges uniformly inside the disk $D$ to some function $f \in H(D)$.

Let us prove that $f \in \tilde{\Pi}_{q}$. We have:

$$
\begin{aligned}
\int_{0}^{1} \int_{-\pi}^{\pi}\left(\ln ^{+}\left|f\left(r e^{i \theta}\right)\right|\right)^{q} d \theta d r & \leqslant \int_{0}^{1} \int_{-\pi}^{\pi}\left(\ln \left(1+\left|f\left(r e^{i \theta}\right)\right|\right)^{q} d \theta d r\right. \\
& \leqslant \int_{0}^{1} \int_{-\pi}^{\pi} \ln ^{q}\left(1+\left|f\left(r e^{i \theta}\right)-f_{n}\left(r e^{i \theta}\right)\right|+\left|f_{n}\left(r e^{i \theta}\right)\right|\right) d \theta d r
\end{aligned}
$$

Since for all $a>0, b>0$ the inequality $(a+b)^{q} \leqslant\left(a^{q}+b^{q}\right)$ holds for $0<q<1$ and $(a+b)^{q} \leqslant 2^{q}\left(a^{q}+b^{q}\right)$ as $q>1$, by the latter estimate we find:

$$
\int_{0}^{1} \int_{-\pi}^{\pi}\left(\ln ^{+}\left|f\left(r e^{i \theta}\right)\right|\right)^{q} d \theta \leqslant \int_{0}^{1} \int_{-\pi}^{\pi}\left[\ln ^{q}\left(1+\left|f\left(r e^{i \theta}\right)-f_{n}\left(r e^{i \theta}\right)\right|\right)+\ln ^{q}\left(1+\left|f_{n}\left(r e^{i \theta}\right)\right|\right)\right] d \theta d r \leqslant \text { const. }
$$


Hence, $\tilde{\Pi}_{q}$ is complete.

c) If $f, f_{n} \in \Pi_{q}$ and $\rho\left(f_{n}, f\right) \rightarrow 0, n \rightarrow+\infty$, then for each $\beta \in \mathbb{C} \rho\left(\beta f_{n}, \beta f\right) \rightarrow 0, n \rightarrow+\infty$.

For $|\beta|<1$, the property follows immediately. Let $|\beta|>1$. We can suppose that $\beta>1$. Since the sequence $\left\{f_{n}\right\}$ converges. it is fundamental. But as it has been established above, this implies the uniform convergence of this sequence inside $D$.

Since for each $\beta \geqslant 1$ and $x \geqslant 0$ the estimate $(1+\beta x) \leqslant(1+x)^{\beta}$ holds, we have

$$
\begin{aligned}
\rho\left(\beta f_{n}, \beta f\right) & =\int_{0}^{1} \int_{-\pi}^{\pi} \ln ^{q}\left(1+\beta\left|f_{n}\left(r e^{i \theta}\right)-f\left(r e^{i \theta}\right)\right|\right) d \theta d r \\
& \leqslant \beta^{q} \int_{0}^{1} \int_{-\pi}^{\pi} \ln ^{q}\left(1+\left|f_{n}\left(r e^{i \theta}\right)-f\left(r e^{i \theta}\right)\right|\right) d \theta d r=\beta^{q} \rho\left(f_{n}, f\right),
\end{aligned}
$$

and this implies property c).

d) If $\beta_{n}, \beta \in \mathbb{C}$ and $\beta_{n} \rightarrow \beta$, then $\rho\left(\beta_{n} f, \beta f\right) \rightarrow 0, n \rightarrow+\infty$ for each function $f \in \tilde{\Pi}_{q}$.

This property is due to the inequality

$$
\ln \left(1+\left|\beta_{n}-\beta\right||f|\right) \leqslant \ln (1+|f|)+\ln \left(1+\left|\beta_{n}-\beta\right|\right) .
$$

The proof is complete.

Lemma 3.3. Let a sequence of complex numbers $\left\{\lambda_{k}\right\}_{k=1}^{+\infty}$ satisfy the following condition:

$$
\left|\lambda_{k}\right|=O\left(\exp \left(-c_{k} k^{\frac{2}{2+q}}\right)\right), \quad k \rightarrow+\infty
$$

for an arbitrary positive sequence $\left\{c_{k}\right\}_{k=1}^{+\infty}, c_{k} \downarrow 0, k \rightarrow+\infty$. Then there exists a number $c>0$ such that for all $k \in \mathbb{N}$ condition $(3.2)$ is satisfied.

The proof of this lemma reproduces the arguing from the proof of Lemma 1 [27] with the exponent $\frac{2}{2+q}$.

Lemma 3.4. Let

$$
g(z)=\exp \frac{c}{(1-z)^{\frac{2}{q}}}, \quad z \in D
$$

where $0<c<\frac{2}{q}$ and $\sum_{n=1}^{+\infty} a_{n}(c) z^{n}$ is the Taylor series of the function $g$. Then the estimate holds:

$$
\left|a_{n}(c)\right| \geqslant \exp \left(c^{\frac{q}{2+q}} \cdot n^{\frac{2}{2+q}}\right) .
$$

The way of proving this lemma reproduces the arguing from the thesis of the author [7] with the exponent $\frac{2}{q}$ and goes back to S.N. Mergelyan [5].

As it has been showed above, the convergence $\rho\left(f_{n}, f\right) \rightarrow 0, n \rightarrow+\infty$ implies the uniform convergence of the sequence of functions $f_{n}(z)$ to the function $f(z)$ in $D$. Therefore, if $f_{n}(z)=$ $\sum_{k=0}^{+\infty} a_{k}^{(n)} z^{k}$ and $f(z)=\sum_{k=0}^{+\infty} a_{k} z^{k}$, then $a_{k}^{(n)} \rightarrow a_{k}, n \rightarrow+\infty$.

Let $X$ be an $F$-space formed by the complex sequences $\left\{b_{k}\right\}_{k}$ such that the convergence of the sequence $\beta^{(n)}=\left\{b_{k}^{(n)}\right\}$ to $\beta=\left\{b_{k}\right\}$ as $n \rightarrow+\infty$ means the component-wise convergence $b_{k}^{(n)} \rightarrow b_{k}, n \rightarrow+\infty, k=0,1,2, \ldots$

We consider the coefficient multiplier $\Lambda=C M\left(\tilde{\Pi}_{q}, X\right)$. By Lemma 3.1, $\Lambda$ is a closed operator. Therefore, by the closed graph theorem [11], the operator $\Lambda$ is continuous and it maps bounded sets in the class $\tilde{\Pi}_{q}$ into bounded sets in the class $X$.

Now are in position to prove Theorem 3.1 
Proof. Let $\Lambda=\left\{\lambda_{k}\right\}_{k=1}^{+\infty}$ be a multiplier from the class $\tilde{\Pi}_{q}$ into the Hardy class $H^{p}(0<p \leqslant \infty)$. We are going to prove that there exists $c>0$ such that estimate (3.1) is satisfied, that is,

$$
\left|\lambda_{k}\right|=O\left(\exp \left(-c k^{\frac{2}{2+q}}\right)\right), \quad k \rightarrow+\infty .
$$

According to Lemma 3.3 , it is sufficient to show that the sequence $\Lambda$ satisfies condition (3.2) for an arbitrary infinitesimal sequence $\left\{c_{k}\right\}_{k=1}^{+\infty}$.

Suppose that we are given an arbitrary positive infinitesimal sequence $\left\{c_{k}\right\}_{k=1}^{+\infty}$. We consider an auxiliary sequence $\left\{c^{\prime}{ }_{k}\right\}_{k=1}^{+\infty}$,

$$
c^{\prime}{ }_{k}=\min \left(\frac{1}{2}, \max \left(k^{-\frac{1}{q}}, c_{k}\right)\right), \quad k=1,2, \ldots
$$

If condition (3.2) is satisfied for this sequence, it remains true also for the sequence $\left\{c_{k}\right\}_{k=1}^{+\infty}$. This is why we can suppose that the terms in the sequence $\left\{c_{k}\right\}_{k=1}^{+\infty}$ satisfy the following condition:

$$
k^{-\frac{1}{q}} \leqslant c_{k} \leqslant \frac{1}{2}
$$

for all $k=1,2, \ldots$. In the class $\tilde{\Pi}_{q}$ we consider the sequence of the functions satisfying the assumptions of Lemma 3.4 :

$$
f_{k}(z)=g\left(r_{k} z\right)=\exp \frac{c_{k}}{\left(1-r_{k} z\right)^{\frac{2}{q}}}, \quad k=1,2, \ldots,
$$

where the sequence $\left\{r_{k}\right\}_{k=1}^{+\infty}$ is such that $r_{k} \rightarrow 1-0, k \rightarrow+\infty$, and

$$
1-\frac{1}{k} \leqslant r_{k} \leqslant 1-\exp \left(-\left(\frac{\gamma_{k}}{c_{k}}\right)^{q}\right), \quad k=1,2, \ldots
$$

Here $\left\{\gamma_{k}\right\}_{k=1}^{+\infty}$ is a positive infinitesimal sequence such that $c_{k}=o\left(\gamma_{k}\right), \quad k \rightarrow+\infty$.

Let us confirm that $f_{k} \in \tilde{\Pi}_{q}$. We have:

$$
\begin{aligned}
\int_{0}^{1} \int_{-\pi}^{\pi}\left(\ln ^{+}\left|f_{k}\left(r e^{i \theta}\right)\right|\right)^{q} d \theta d r & =\int_{0}^{1} \int_{-\pi}^{\pi}\left(\ln ^{+}\left|\exp \frac{c_{k}}{\left(1-r_{k} r e^{i \theta}\right)^{\frac{2}{q}}}\right|\right)^{q} d \theta d r \\
& \leqslant \int_{0}^{1} \int_{-\pi}^{\pi} \frac{c_{k}^{q}}{\left|1-r_{k} r e^{i \theta}\right|^{2}} d \theta \leqslant \int_{0}^{1} \frac{c_{k}^{q}}{\left(1-r_{k} r\right)} d r=c_{k}^{q} \ln \frac{1}{1-r_{k}}=\gamma_{k}^{q} .
\end{aligned}
$$

We are going to show that $\left\{f_{k}\right\}_{k=1}^{+\infty}$ is a bounded sequence in the class $\tilde{\Pi}_{q}$, that is, there exists a real number $0<\lambda<1$ such that for all natural $k$ the inequality $\rho\left(\lambda f_{k}, 0\right)<\varepsilon$ holds, where $\varepsilon$ is a fixed positive number, see [11. In order to do this, first we are going to check the inequality

$$
\ln (1+|\lambda||g|) \leqslant\left(\ln (1+|\lambda|)+\ln ^{+}|g|\right) .
$$

Indeed, if $|g| \leqslant 1$, then $|\lambda||g| \leqslant|\lambda|$ and estimate (3.8) follows immediately.

If $|g| \geqslant 1$, then

$$
\ln (1+|\lambda||g|) \leqslant \ln (|g|+|\lambda||g|) \leqslant \ln (1+|\lambda|)+\ln ^{+}|g| .
$$

Now let us prove the inequality $\rho\left(\lambda f_{k}, 0\right)<\varepsilon$. Let $0<q<1$; for $q>1$ the proof is the same. We have

$$
\rho\left(\lambda f_{k}, 0\right)=\int_{0}^{1} \int_{-\pi}^{\pi} \ln ^{q}\left(1+\left|\lambda f_{k}\left(r e^{i \theta}\right)\right|\right) d \theta d r \leqslant 2 \pi\left(\ln ^{q}(1+|\lambda|)+\left(\gamma_{k}\right)^{q}\right) .
$$


Since $\gamma_{k}=o(1), k \rightarrow+\infty$, the for each $\varepsilon>0$ there exists a number $k_{0} \in \mathbb{N}$ such that for all $k \geqslant k_{0}$ the inequality

$$
\gamma_{k}<\sqrt[q]{\frac{\varepsilon}{4 \pi}}
$$

holds true. Choosing $\lambda_{k_{0}}$, such that

$$
\ln \left(1+\left|\lambda_{k_{0}}\right|\right)<\sqrt[q]{\frac{\varepsilon}{4 \pi}}
$$

we see that starting from the number $k_{0}$, all elements of the sequence $\left\{f_{k}\right\}$ are contained in the ball of the radius $\varepsilon$.

Since $\tilde{\Pi}_{q}$ is an $F$-space, then for all indices $k<k_{0}$ there exists a positive number $\lambda_{k}$ such that for all $\lambda \in \mathbb{C}$ with $|\lambda| \leqslant \lambda_{k}$ the inequality $\rho\left(\lambda f_{k}, 0\right)<\varepsilon$ holds. Letting $\lambda_{0}=\min \left(\lambda_{1}, \lambda_{2}, \ldots, \lambda_{k_{0}}\right)$, we get that for $|\lambda| \leqslant \lambda_{0}$ the entire sequence $\left\{f_{k}\right\}$ is contained in the ball of the radius $\varepsilon$, that is, $\rho\left(\lambda f_{k}, 0\right)<\varepsilon$.

By the arbitrariness of $\varepsilon$ we conclude that $\left\{f_{k}\right\}$ is a bounded sequence in the class $\tilde{\Pi}_{q}$.

Thus, we have proved that for all natural $k$ the sequence of the functions $\left\{f_{k}\right\}_{k=1}^{+\infty}$ is bounded in $\tilde{\Pi}_{q}$ and hence, the coefficient multiplier $\Lambda\left(f_{k}\right)$ is bounded in the class $H^{p}$.

We have:

$$
\left\|\Lambda\left(f_{k}\right)\right\|_{H^{p}} \leqslant C, \quad C>0 .
$$

We fix $k \in \mathbb{N}$. If $f_{k}(z)=\sum_{n=0}^{+\infty} a_{n}^{(k)} z^{n} \in \tilde{\Pi}_{q}$, then $\Lambda\left(f_{k}\right)(z)=\sum_{n=0}^{+\infty} \lambda_{n} a_{n}^{(k)} z^{n} \in H^{p}$, and hence, [19]

$$
\begin{aligned}
& \left|\lambda_{n} a_{n}^{(k)}\right| \leqslant c_{p}\left\|\Lambda\left(f_{k}\right)\right\|_{H^{p}} n^{\frac{1}{p}-1} \quad \text { as } \quad 0<p<1, \\
& \left|\lambda_{n} a_{n}^{(k)}\right| \leqslant c_{p}\left\|\Lambda\left(f_{k}\right)\right\|_{H^{p}} \quad \text { as } \quad 1 \leqslant p \leqslant \infty
\end{aligned}
$$

which implies

$$
\begin{aligned}
& \left|\lambda_{n} a_{n}^{(k)}\right| \leqslant C c_{p} n^{\frac{1}{p}-1} \quad \text { as } \quad 0<p<1, \\
& \left|\lambda_{n} a_{n}^{(k)}\right| \leqslant C c_{p} \quad \text { as } \quad 1 \leqslant p \leqslant+\infty,
\end{aligned}
$$

where $c_{p}$ is a positive constant depending on the parameter $p$.

Since $f_{k}(z)=g\left(r_{k} z\right)$, then $a_{n}^{(k)}=a_{n}\left(c_{k}\right) r_{k}^{n}$. In accordance with Lemma 3.4 .

$$
\left|a_{n}^{(k)}\right| \geqslant r_{k}^{n} \exp \left(c_{k}^{\frac{q}{2+q}} n^{\frac{2}{2+q}}\right) .
$$

Taking into consideration inequality (3.7), we obtain:

$$
\left|a_{k}^{(k)}\right| \geqslant\left(1-\frac{1}{k}\right)^{k} \exp \left(c_{k}^{\frac{q}{2+q}} n^{\frac{2}{2+q}}\right) .
$$

By (3.9), 3.11) we conclude:

$$
\left|\lambda_{k}\right| \leqslant C c_{p}^{\prime}\left(1-\frac{1}{k}\right)^{-k} k^{\frac{1}{p}-1} \exp \left(c_{k}^{\frac{q}{2+q}} n^{\frac{2}{2+q}}\right),
$$

and in view of estimate 3.5 we get:

$$
\left|\lambda_{k}\right| \leqslant \widetilde{C} \exp \left(c_{k}^{\frac{q}{2+q}} n^{\frac{2}{2+q}}\right) .
$$

Applying Lemma 3.3 , by inequality $(3.12)$ we conclude that estimate $(3.1)$ is valid. In the same way, as $1 \leqslant p<+\infty$, by $3.10,3.12$ we arrive at the desired estimate. 
We proceed to proving the inverse statement of Theorem 3.1. Let a $\Lambda=\left\{\lambda_{k}\right\}_{k=1}^{+\infty}$ satisfies condition (3.1) of the theorem and $f \in \tilde{\Pi}_{q}, f(z)=\sum_{k=0}^{+\infty} a_{k} z^{k}$. It follows from Theorem 2.2 that

$$
\left|a_{k}\right| \leqslant C_{1} \exp \left(\varepsilon_{k} k^{\frac{2}{2+q}}\right), \quad \varepsilon_{k} \downarrow 0, \quad k \rightarrow+\infty .
$$

We choose an index $k_{0}$ so that $\varepsilon_{k}<\frac{c}{2}$ for all $k \geqslant k_{0}$ and we get:

$$
\left|\lambda_{k} a_{k}\right| \leqslant C_{2} \exp \left(-\frac{c}{2} k^{\frac{2}{2+q}}\right) \text {. }
$$

Since the series $\sum_{k=0}^{+\infty} \exp \left(-\frac{c}{2} k^{\frac{2}{2+q}}\right)$ converges, then $\Lambda(f)(z) \in H^{p}$. The proof is complete.

Remark 3.1. We note that the way of proving Theorem 3.1 goes back to work [27] by N. Yanagihara. Theorem 3.1 remains true also if the Hardy class is replaced by the Bergman class $A_{\alpha}^{p}$,

$$
A_{\alpha}^{p}:=\left\{f \in H(D): \int_{0}^{1} \int_{0}^{2 \pi}(1-r)^{\alpha}\left|f\left(r e^{i \theta}\right)\right|^{p} d \theta r d r<+\infty\right\}, \quad p>0, \quad \alpha>-1,
$$

or by the class $\tilde{\Pi}_{q^{\prime}}, 0<q<q^{\prime}$.

Remark 3.2. An immediate corollary of proven Theorem 3.1 is the statement on sharpness of the estimates obtained in Theorems 2.2 and 2.1. The proof of this fact is made in the same way as in work by R. Meštrović, see [1, Cors. 9.24, 9.26].

\section{ACKNOWLEDGMENTS}

The author expresses her sincere gratitude to her scientific supervisor professor F.A. Shamoyan for useful discussions.

\section{BIBLIOGRAPHY}

1. V.I. Gavrilov, A.V. Subbotin, D.A. Efimov. Boundary properties of analytic functions (further contribution). Izd-vo MGU, Moscow (2012).

2. R. Nevanlinna. Eindeutige analytische Funktionen. Springer-Verlag, Berlin (1953). [Analytic functions. Springer-Verlag, Berlin (1970).]

3. M.A. Evgrafov. The behavior of a power series for functions of the class $H_{\delta}$ on the boundary of the disk of convergence // Izv. AN SSSR. Ser. Mat. 16:5, 481-492 (1952). (in Russian).

4. I.I. Privalov. Boundary properties of single-valued analytic functions. Izd-vo MGU, Moscow (1941). (in Russian).

5. I.I. Privalov. Boundary properties of analytic functions. GITTL, Moscow. (in Russian).

6. E.G. Rodikova. On estimates for expansion coefficients for certain classes of functions analytic in a disk // in Proceedings of the VI Petrozavodsk International Conference "Complex Analysis and Applications", Petrozavodsk. 64-69 (2012). (in Russian).

7. E.G. Rodikova. Factorization, characterization of root sets, and questions of interpolation in weighted spaces of analytic functions. PhD thesis, Bryansk (2014). (in Russian).

8. E.G. Rodikova. On properties of zeros of functions from Privalov classes in disk // Uchen. Zapis. Bryansk. Gosud. Univr. 4, 19-22 (2019). (in Russian).

9. E.G. Rodikova. On interpolation sequences in the Privalov space // in Proceedings of International Scientific Conference "Complex analysis, mathematical physics and nonlinear equations", Ufa. 52-53 (2020). (in Russian).

10. E.G. Rodikova, V.A. Bednazh. On interpolation for the Privalov classes in a disk // Sibir. Elekt. Matem. Izv. Siber. 16, 1762-1775 (2019). (in Russian).

11. W. Rudin. Functional analysis. McGraw-Hill Book Comp., New York (1973). 
12. F.A. Shamoyan. Parametric representation and description of the root sets of weighted classes of functions holomorphic in the disk // Sibir. Matem. Zhurn. 40:6, 1422-1440 (1999). [Siberian Math. J. 40:6, 1211-1229 (1999).]

13. F.A. Shamoyan. Weighted spaces with mixed norm. Izd. Bryanskogo Gosud. Univ., Bryansk (2014). (in Russian).

14. F.A. Shamoyan. Some properties of the zero sets of a function from Privalov's class in a disk // Zap. Nauch. Semin. POMI. 480, 199-205 (2019). (in Russian).

15. F.A. Shamoyan, E.N. Shubabko. A class of functions holomorphic in the disk // Zap. Nauch. Semin. POMI. 282, 244-255 (2001). [J. Math. Sci. 120:5, 1784-1790 (2004).]

16. F.A. Shamoyan, V.A. Bednazh, O.V. Prihod'ko. On zero sets of certain weighted classes of analytic functions in a disk // Vestnik Bryansk. Gosud. Univ. 4 (2008), 85-92. (in Russian).

17. S.V. Shvedenko. Hardy classes and related spaces of analytic functions in the unit circle, polydisc and ball // Itogi Nauki i Tekhniki. Ser. Mat. Anal. 23, 3-124 (1985). [J. Soviet Math. 39:6, 3011-3087 (1987).]

18. S.V. Shvedenko. The rate of growth and Taylor coefficients of functions of Nevanlinna's area class N // Izv. Vuzov. Mat. 6, 40-43 (1986). [ Soviet Math. (Iz. VUZ). 30:6, 56-60 (1986).]

19. P.L. Duren. Theory of $H^{p}$ spaces. Academic Press, New York (1970).

20. O. Frostman. Sur les produits des Blaschke // Kungl. Fysiografiska Sällskapets i Lund Förhandlingar, Proa. Roy. Physiog. Soa. Lund. 12:15, 169-182 (1942).

21. W.K. Hayman, B. Korenblum. A critical growth rate for functions regular in a disk // Michigan Math. J. 27:1, 21-30 (1980).

22. M. Pavlović. Introduction to function spaces in the disk. Matematički Institut SANU, Belgrade (2004).

23. E.G. Rodikova. Coefficient multipliers for the Privalov class in a disk // Zhurn. SFU. Ser. Matem. Fiz. 11:6, 723-732 (2018).

24. E.G. Rodikova. Multiple interpolation in the Privalov classes in a disk // Filomat. 35:1, 271-286 (2021).

25. E.G. Rodikova, F.A. Shamoyan. On the differentiation in the Privalov classes // Zhurn. SFU. Ser. Matem. Fiz. 13:5, 622-630 (2020).

26. M. Stoll. Mean growth and Taylor coefficients of some topological algebras of analytic functions // Ann. Polon. Math. 35:2, 139-158 (1977).

27. N. Yanagihara. Multipliers and linear functionals for the class $N^{+} / /$Trans. Amer. Math. Soc. 180, 449-461 (1973).

28. N. Yanagihara. Mean growth and Taylor coefficients of some classes of functions // Ann. Polon. Math. 30, 37-48 (1974).

Evgenia Gennadievna Rodikova,

Bryansk State University,

Bezhitskaya str. 14,

241050, Bryansk, Russia

E-mail: evheny@yandex.ru 